\title{
Essential newborn care after home delivery in Nepal
}

\author{
MATS MÅLQVIST ${ }^{1}$, ASHA PUN ${ }^{2}$ \& ASHISH KC $\mathrm{KC}^{1,2}$ \\ ${ }^{1}$ International Maternal and Child Health, Department of Women's and Children's Health, Uppsala University, Sweden, \\ and ${ }^{2}$ UN Health Section, UNICEF Nepal Country Office, UN House, Pulchowk, Nepal
}

\begin{abstract}
Aims: Postnatal care of the newborn is essential in order to reduce neonatal mortality. Nepal has made great efforts to improve maternal and child health by focusing on accessibility and outreach over the past decades. This study aims to examine trends, over the past decade, in levels and equity of facility delivery rates and the provision of newborn care after home delivery in Nepal. Methods: Household-level data from the Demographic Health Surveys (DHS) 2006 and 2011 and the Multiple Indicator Cluster Survey (MICS5) from 2014 performed in Nepal was sourced for the study. Coverage rates of facility delivery and newborn care after home delivery were calculated and logistic regression models were used to ascertain inequity. Results: Home delivery rate dropped from $79.2 \%$ in 2006 to $46.5 \%$ in 2014 , a development showing an inequitable distribution, with a larger share of better-off families shifting to facility delivery. For those who still delivered at home there was an increased rate of early initiation of breastfeeding and adequate temperature control, but only $2.2 \%$ of women delivering at home received a home visit by a health professional in the first week of delivery. No inequity in receiving newborn care after home delivery could be detected. Conclusions: There have been significant improvements in facility delivery rates over the last 10 years in Nepal and postnatal care at home has improved. There is, however, an alarmingly low level of home visits during an infant's first week.
\end{abstract}

Key Words: Postnatal care, newborn, home delivery, Nepal, equity, socioeconomic determinants

\section{Introduction}

Postnatal care is crucial for the survival and good health of newborns. Good hygiene, knowledge of danger signs, temperature control and early initiation of breastfeeding are all interventions needed to reduce the risk of neonatal mortality. In 2013 WHO updated its guidelines on postnatal care of the newborn, incorporating research findings produced in recent years [1]. Worth noting in the updated guidelines are, for example, the emphasis on early home visits to babies delivered at home $[2,3]$ and the use of chlorhexidine for umbilical cord care in settings with high neonatal mortality [4-6], both interventions strongly recommended based on evidence. The implementation of quality postnatal care is, however, a challenge, both in health facilities as well as in the community. Facility delivery should be encouraged for many reasons, such as the ability to provide adequate postnatal care and the opportunity to educate families on how to take care of their newborns upon returning home. In settings where home delivery is still prevalent and the shift to facility delivery is slow, however, it becomes important not only to focus on getting pregnant women to facilities, but also to ensure quality postnatal care in the home [3].

Nepal has made great strides towards improved maternal and child health through focusing on accessibility and outreach over the past decades $[7,8]$. Maternal mortality has declined from 548/100,000

Correspondence: Mats Målqvist, International Maternal and Child Health (IMCH), Department of Women's and Children's Health, Uppsala University, SE-751 85 Uppsala, Sweden. E-mail: mats.malqvist@kbh.uu.se

(Accepted 15 November 2016)

(C) Author(s) 2016

Reprints and permissions: sagepub.co.uk/journalsPermissions.nav DOI: $10.1177 / 1403494816683572$

journals.sagepub.com/home/sjp 
deliveries in year 2000 to $258 / 100,000$ in 2015 [9], and under-5 mortality has declined from $81 / 1000$ to $36 / 1000$ live births over the same period [9]. There are, however, indications that the neonatal mortality reduction is stagnating, thus constituting a larger part of overall child mortality. There is, therefore, a need to focus more on essential newborn care as a means to further reduce overall child mortality. Despite recent efforts to increase facility delivery rates in Nepal, a large fraction of deliveries take place at home, and the utilization of postnatal care services and practices is still low $[10,11]$. Difficult terrain and a weak health system pose great challenges to the provision of facility delivery for all. The Nepalese authorities have recognized this, and major investments in community-based services have been made in tandem with strengthening health facilities [8].

Not only do geographical difficulties influence the provision of adequate newborn care; socio-economic barriers are also present [12]. A recent study identified various socio-economic factors associated with postnatal care provision, demonstrating that the better-off, the better educated and those living in urban areas were more likely have a facility delivery and to practice appropriate immediate postnatal care [7]. It also found that health facility delivery was positively associated with the provision and utilization of postnatal care, as have other studies that have found a positive association between facility delivery and early initiation of breastfeeding [13] and thermal control [14], both important components of newborn care. These associations highlight the need to improve postnatal care provision after home delivery. The study also stresses the importance of investigating the dynamics of how structural determinants influence the provision of postnatal care in order to target interventions and follow progress. The aims of this present study were, thus, to examine trends in levels and equity in relation to facility delivery rates and the provision of newborn care after home delivery in Nepal over the past decade.

\section{Methods}

Data from DHS 2006 and 2011 and MICS5 from 2014 were utilized for the study. Sampling methodologies for the respective surveys have been described elsewhere [15-17]. DHS data has been accessed with permission from ORC Macro, and MICS 2014 has been accessed and analysed with permission from UNICEF. A sub-sample of the surveys including women who had given birth at home within two (MICS) and five (DHS) years prior to the survey data was then used for analysis of trends in postnatal care provision. The most recent delivery recorded was used for analysis; thus, only one delivery per woman was included.

\section{Variables of interest}

The following primary outcome variables were chosen, guided by availability of data and the most recent WHO recommendations on postnatal care [1]:

1. Early initiation of breastfeeding: Early initiation of breastfeeding is considered an important intervention, and as a gold standard, breastfeeding should be initiated immediately or within the first hour after delivery.

2. Dry cord care: According to WHO guidelines, application of chlorhexidine to the umbilical cord should be done daily for babies delivered at home. If there is no chlorhexidine available, the cord should be left dry and any other application should be discouraged. A rapid assessment of the survey data material indicated that chlorhexidine had only been used in a very few cases; thus, dry cord care was chosen as a primary outcome variable.

3. Temperature control: Keeping the baby warm is a key feature of postnatal care involving procedures such as drying the baby, skin to skin care, covering the baby and delayed bathing. For this study, indicators of whether the baby was dried and cleaned before the placenta was delivered and whether the baby was bathed within the first 24 hours or not were used as variables for temperature control.

4. Home visit by health professional during the first week after delivery: Recommendations for home deliveries are that the mother and baby should receive a home visit by a health professional, preferably within 24 hours after delivery and then on day three. For this study, a home visit within the first week after delivery was considered a primary outcome.

In order to obtain comprehensive outcome measures, a composite score for each woman was calculated based on how many of the above interventions were received, thus generating a score of $0-4$. After an initial assessment, a dichotomization of women receiving two or more of the interventions and women receiving none or only one was calculated and used as the dependent variable in logistic regression analyses.

The primary outcomes were stratified by the independent variables wealth, maternal education level and living area. Ethnicity and caste play a major role in Nepal, but data on these indicators was not 
Table I. Characteristics of women who had given birth at home within two years preceding survey in Nepal.

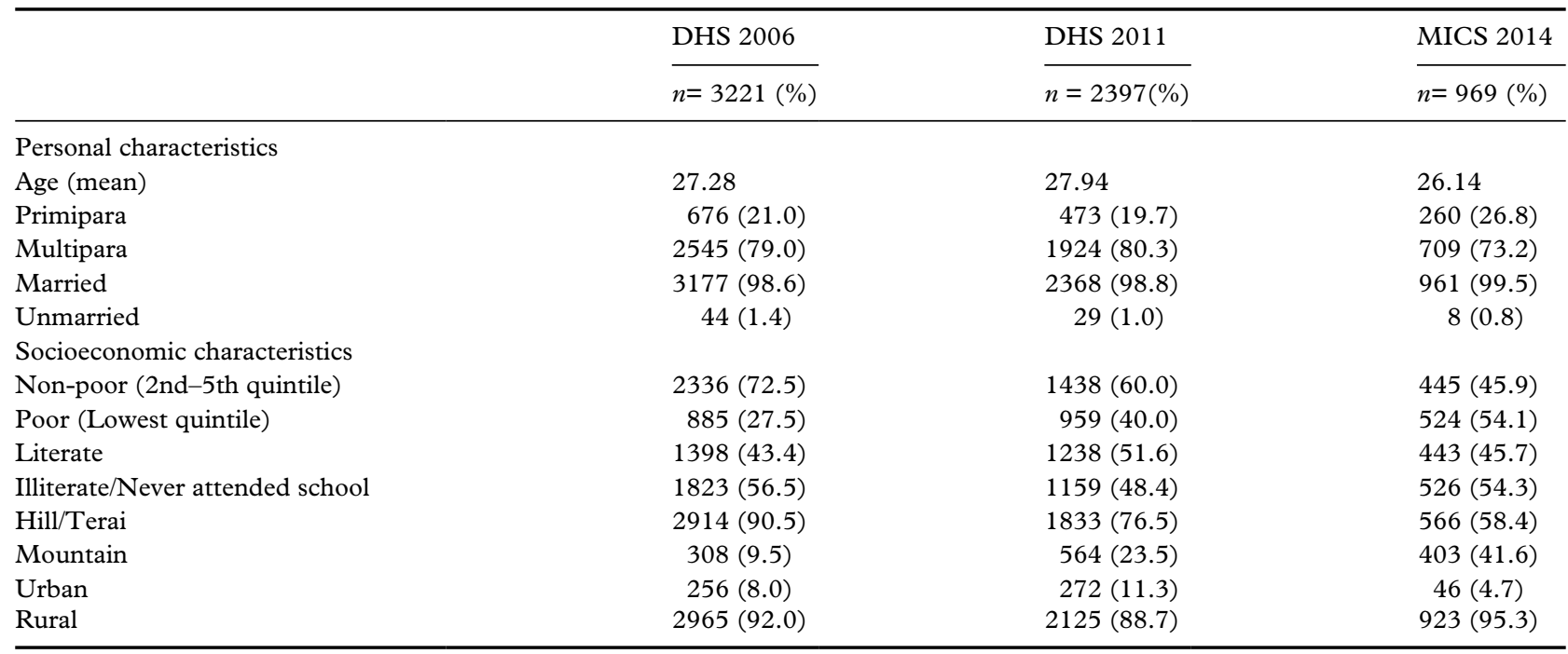

collected stringently in the different surveys and was therefore omitted. Wealth, or economic status, was defined through a pre-set wealth index based on assets and calculated by principal components analysis as defined in the respective survey methodologies [18]. Mother's education level was defined as the mother being illiterate or literate. Given the implications of location for home visits and accessibility, especially in a country like Nepal with many mountainous and inaccessible areas, place of residence was also included in the analysis, divided into urban or rural dwelling and whether the family was living in the mountains or not.

\section{Data analysis}

The prevalence of the selected variables for postnatal care was calculated and divided by the structural determinants described above. Chi-square test was used to detect group differences and a $p$-value of $<.05$ was considered significant. A multivariable logistic regression analysis was then applied with all independent variables included. Adjusted odds ratios and $95 \%$ confidence intervals were calculated. All analyses were performed in SPSS 20.0.

\section{Results}

A total of 4066 (DHS 2006), 4079 (DHS 2011) and 2086 (MICS 2014) deliveries were sourced from the three survey data sets. Of these, 3221, 2397 and 969 deliveries, respectively, occurred at home, showing a declining home delivery rate of $79.2 \%, 58.5 \%$ and $46.5 \%$ in the respective surveys $(p<.001$ for all group comparisons) (Table I). Mean age of the respondents varied between 27.94 and 26.14. A higher share of women were primiparas in the MICS 2014 survey $(26.5 \%)$ compared with the two previous surveys $(p<.001)$.

With the declining home delivery rate, a larger proportion of women delivering at home were women from poor households, indicating that the shift from home to facility deliveries has mainly happened within the better-off segments of society. It can also be noted that among home deliveries there was an increase over time in the proportion living in mountainous and rural areas, affirming that the trend towards more facility delivery has mainly happened in the more accessible and affluent parts of society. A similar trend could, however, not be seen among illiterate women, for whom there is no difference over time, comparing data from 2006 and 2014, in the proportions of home deliveries $(p=.20)$.

Few women delivering at home received a home visit by a trained health provider during the first week. In 2006 only $4.9 \%$ of babies delivered at home received a home visit during the first week, a percentage that was further reduced to $2.2 \%$ in 2014 $(p=.001)$ (Table II). There was, however, improvement in early initiation of breastfeeding, from 34.3\% to $51.8 \%$ over time $(p<.001)$ and temperature control, from $8.3 \%$ in 2006 to $34.6 \%$ in 2014 . A contrary pattern was shown for dry cord care, with a decline from $74.0 \%$ to $50.5 \%$. Dry cord care being the second best option for babies delivered at home, it might be assumed that the first and recommended option with chlorhexidine would explain the decrease in dry cord practices, but the share of babies receiving chlorhexidine was minimal and could not explain the decrease in dry cord care practices, thus 
Table II. Postnatal care in home deliveries in Nepal over time.

\begin{tabular}{|c|c|c|c|c|c|c|}
\hline & \multicolumn{2}{|l|}{2006} & \multicolumn{2}{|l|}{2011} & \multicolumn{2}{|l|}{2014} \\
\hline & $N$ & $\%$ & $N$ & $\%$ & $N$ & $\%$ \\
\hline $\begin{array}{l}\text { Baby's health checked at least once in first week } \\
\text { (day 1-6) by trained health professional (before } \\
\text { leaving hospital or at home) }\end{array}$ & $158 / 3221$ & 4.9 & $91 / 2395$ & 3.8 & $21 / 946$ & 2.2 \\
\hline $\begin{array}{l}\text { Early initiation of breastfeeding } \\
\text { (immediately or within } 1 \text { hour) }\end{array}$ & $1094 / 3191$ & 34.3 & $951 / 2364$ & 40.2 & $495 / 956$ & 51.8 \\
\hline Dry cord care & $2361 / 3191$ & 74.0 & $1412 / 2324$ & 60.8 & $465 / 941$ & 50.5 \\
\hline Chlorhexidine on cord stump & $\mathrm{N} / \mathrm{A}^{\mathrm{a}}$ & & $11 / 2397$ & 0.5 & 9/941 & 1.9 \\
\hline Improper application on cord & $\mathrm{N} / \mathrm{A}^{\mathrm{a}}$ & & $901 / 2324$ & 38.8 & $445 / 941$ & 47.3 \\
\hline Proper cord care & $\mathrm{N} / \mathrm{A}^{\mathrm{b}}$ & & $1423 / 2324$ & 61.2 & $496 / 941$ & 52.7 \\
\hline Delayed bathing ( $>24 \mathrm{~h}$ after delivery) & $503 / 3198$ & 15.7 & $507 / 2385$ & 21.3 & $440 / 958$ & 45.9 \\
\hline Baby dried and cleaned before placenta delivered & $1380 / 3220$ & 42.8 & $1420 / 2397$ & 59.2 & $708 / 955$ & 74.1 \\
\hline Adequate temperature control & $263 / 3181$ & 8.3 & $361 / 2368$ & 15.2 & $327 / 945$ & 34.6 \\
\hline \multicolumn{7}{|l|}{$\begin{array}{l}\text { Quality postnatal care (Composite score of early } \\
\text { initiation of breastfeeding, dry cord care, adequate } \\
\text { temperature control and health check performed) }\end{array}$} \\
\hline 0 out of 4 & $471 / 3142$ & 15.0 & $471 / 2279$ & 20.7 & $140 / 869$ & 16.1 \\
\hline 1 out of 4 & $1657 / 3142$ & 52.7 & $1042 / 2279$ & 45.7 & $330 / 869$ & 38.0 \\
\hline 2 out of 4 & $894 / 3142$ & 28.4 & $618 / 2279$ & 27.1 & $316 / 869$ & 36.2 \\
\hline 3 out of 4 & $119 / 3142$ & 3.8 & $145 / 2279$ & 6.4 & $84 / 869$ & 9.7 \\
\hline 4 out of 4 & $2 / 3142$ & 0.1 & $3 / 2279$ & 0.1 & $0 / 869$ & 0.0 \\
\hline
\end{tabular}

avariable not included in data set.

bInsufficient data.

Table III. Multivariate logistic regression displaying adjusted odds ratios for babies delivered at home receiving at least two of four postnatal care interventions (early initiation of breastfeeding, dry cord care, adequate temperature control and/or home visit during first week) in Nepal 2006-2014, adjusted for maternal age.

\begin{tabular}{|c|c|c|c|c|c|c|}
\hline & \multicolumn{2}{|l|}{2006} & \multicolumn{2}{|l|}{2011} & \multicolumn{2}{|l|}{2014} \\
\hline & OR & CI $95 \%$ & OR & CI $95 \%$ & OR & CI $95 \%$ \\
\hline Non-poor (2nd-5th quintile) & Ref & & Ref & & Ref & \\
\hline Poor (Lowest quintile) & 0.76 & $0.64-0.91^{\mathrm{a}}$ & 0.87 & $0.72-1.06$ & 1.11 & $0.84-1.49$ \\
\hline Literate & Ref & & Ref & & Ref & \\
\hline Illiterate & 0.71 & $0.61-0.84^{\mathrm{a}}$ & 0.45 & $0.37-0.55^{\mathrm{a}}$ & 0.82 & $0.61-1.09$ \\
\hline Hill/Terai & Ref & & Ref & & Ref & \\
\hline Mountain & 0.98 & $0.75-1.28$ & 1.25 & $1.01-1.54^{\mathrm{a}}$ & 1.13 & $0.85-1.51$ \\
\hline Urban & Ref & & Ref & & Ref & \\
\hline Rural & 1.19 & $0.90-1.59$ & 0.93 & $0.70-1.23$ & 1.81 & $0.89-3.67$ \\
\hline
\end{tabular}

CI: confidence interval; OR: odds ratio.

a $p<.05$.

indicating an increase in non-favoured practices for cord care (Table II).

When calculating a composite score of postnatal care based on the variables investigated, there is a trend towards receiving more proper intervention in connection with home delivery. The share of mothers and babies receiving two or more of the four postnatal interventions increased from $32.3 \%$ in 2006 to $46.0 \%$ in $2014(p<.001)$ (Table II). Within the group of home deliveries in the survey performed in 2014, no inequity based on wealth, education or living area could be detected in the likelihood of receiving two or more of the selected interventions (Table III). The previous survey (DHS 2006) revealed a decreased likelihood of receiving two or more of the postnatal interventions for poor women, indicating a closing equity gap in postnatal care for home deliveries since the survey in 2006 .

\section{Discussion}

Our results, based on survey data from three consecutive surveys, reveal large deficits in the provision of quality postnatal care to women delivering at home in Nepal. Despite guidelines stipulating home visits by trained health staff within the first week after delivery[1], preferably already on the first day, and that chlorhexidine should be used once daily on the 
umbilical stump, these interventions have reached only a few of the babies delivered at home. While at the same time there have been substantial improvements in reducing home delivery rates, efforts must be stepped up to provide quality care at home for the large number of women delivering at home in Nepal.

Even if the evidence-based provision of home visits and the application of chlorhexidine have not met expectations, there have been other improvements in essential newborn care provision over the last decade. The rates of early initiation of breastfeeding and adequate temperature control in the home delivery group have increased two and fourfold, respectively, though there is still plenty of room for improvement. Cord care practices seem, on the contrary, to have worsened over the years, with a $50 \%$ reduction in the practice of dry cord care from the 2006 survey to the 2014 survey. What might have caused this large reduction is difficult to ascertain, but it is a trend worth highlighting.

There are some interesting equity dynamics that can be noted in the results. Overall home delivery rate has decreased, and it has previously been shown how this change occurred with an unchanged equity gap over wealth quintiles [19]. Looking at newborn care after home delivery, it can, however, be noted that the improvements seen over time have also resulted in reduced inequity of the interventions. The equity gap between the poor and the better-off and the illiterate and literate mothers that existed in 2006 for essential newborn care could not be found in the survey from 2014. Education has previously been demonstrated to be associated with early initiation of breastfeeding in Nepal [19], but just as with most studies, equity analyses have been performed on the total population, not differentiating between deliveries at a facility and at home. The effect of education, and other structural determinants, could thus be confounded by delivery place, as postnatal care can be assumed to have better coverage at facility level.

There are some methodological limitations worth mentioning. We have compared survey data from two different survey methodologies, the DHS and the MICS. Both survey sampling designs can be considered representative for Nepal at large, even if the sample size in the DHS surveys is about twice the size of the MICS sample. There are also differences in the way questions are designed, and not all information is found in both surveys. We have been limited to utilizing the postnatal care indicators that were similar in the two study designs, even if it meant discarding data that might have shed a different light if only one survey had been analysed. For temperature control and timing of health check-up, more information is available in the data sets but is not comparable between sets. Creating a consolidated score for postnatal care using four indicators as proxy could also be viewed as a blunt method, but we were limited by the availability of comparable data sets.

\section{Conclusion}

Great improvements have been made in providing postnatal care to the most vulnerable segments of society in Nepal. Home delivery rates have decreased over time, and for the shrinking share of babies delivered at home, postnatal care provision has increased overall. There is also evidence of a closing equity gap in essential newborn care in the home delivery group. More efforts are, however, needed, as coverage of postnatal care interventions remains low.

\section{Acknowledgements}

MM and AK conceptualized the study. MM performed statistical analyses and prepared the first draft. All authors contributed to the finalization of the manuscript. This was a secondary analysis of DHS and MICS data. Ethical approval and consent to participate followed the DHS and MICS methodologies and designs. Data is available with permission from http://data.unicef.org and from http://dhsprogram.com/

\section{Declaration of conflicting interests}

The author(s) declared no potential conflicts of interest with respect to the research, authorship, and/ or publication of this article.

\section{Funding}

The author(s) disclosed receipt of the following financial support for the research, authorship, and/or publication of this article: This study was financed by UNICEF Nepal. Data was accessed by permission from DHS and MICS.

\section{References}

[1] World Health Organization. WHO recommendations on postnatal care of the mother and newborn. Geneva: World Health Organization, 2014.

[2] Baqui AH, Ahmed S, El Arifeen S, et al. Effect of timing of first postnatal care home visit on neonatal mortality in Bangladesh: An observational cohort study. BMF 2009;339: b2826.

[3] Gogia S and Sachdev HS. Home visits by community health workers to prevent neonatal deaths in developing countries: A systematic review. Bull World Health Organ 2010;88: 658666B.

[4] Arifeen SE, Mullany LC, Shah R, et al. The effect of cord cleansing with chlorhexidine on neonatal mortality in rural Bangladesh: A community-based, cluster-randomised trial. Lancet 2012;379: 1022-1028. 
[5] Soofi S, Cousens S, Imdad A, et al. Topical application of chlorhexidine to neonatal umbilical cords for prevention of omphalitis and neonatal mortality in a rural district of Pakistan: A community-based, cluster-randomised trial. Lancet 2012;379: 1029-1036.

[6] Mullany LC, Darmstadt GL, Khatry SK, et al. Impact of umbilical cord cleansing with $4.0 \%$ chlorhexidine on time to cord separation among newborns in southern Nepal: A cluster-randomized, community-based trial. Pediatrics 2006;118: 1864-1871.

[7] Khanal V, Adhikari M, Karkee R, et al. Factors associated with the utilisation of postnatal care services among the mothers of Nepal: Analysis of Nepal demographic and health survey 2011. BMC Women's Health 2014;14: 19.

[8] Pradhan YV, Upreti SR, Pratap KCN, et al. Newborn survival in Nepal: A decade of change and future implications. Health Policy Plan 2012;27 Suppl 3: iii57-71.

[9] World Health Organization, UNICEF, UNFPA, World Bank Group and United Nations Population Division Maternal Mortality Estimation Inter-Agency Group. Maternal mortality in 1990-2015 [Nepal]. Geneva:World Health Organization, 2015.

[10] Neupane S and Doku D. Utilization of postnatal care among Nepalese women. Matern Child Health f 2013;17: 19221930.

[11] Karkee R and Khanal V. Postnatal and neonatal care after home birth: A community-based study in Nepal. Women Birth 2016;29: e39-43.

[12] Paudel D, Shrestha IB, Siebeck M, et al. Neonatal health in Nepal: Analysis of absolute and relative inequalities and impact of current efforts to reduce neonatal mortality. $B M C$ Public Health 2013;13: 1239.

[13] Adhikari M, KhanalV, Karkee R, et al. Factors associated with early initiation of breastfeeding among Nepalese mothers: Further analysis of Nepal Demographic and Health Survey, 2011. Int Breastfeed f 2014;9: 21.

[14] Pagel C, Prost A, Hossen M, et al. Is essential newborn care provided by institutions and after home births? Analysis of prospective data from community trials in rural South Asia. BMC Pregnancy Childbirth 2014;14: 99.

[15] Central Bureau of Statistics/Government of Nepal and UNICEF. Nepal Multiple Indicator Cluster Study 2014; Key findings and tables. Kathmandu: Government of Nepal, 2015.

[16] Ministry of Health and Population (MOHP) [Nepal], New ERA and ICF International Inc. Nepal Demographic and Health Survey 2006. Kathmandu, Nepal: Ministry of Health and Population, New ERA, and ICF International, Calverton, Maryland, 2007.

[17] Ministry of Health and Population (MOHP) [Nepal], New ERA and ICF International Inc. Nepal Demographic and Health Survey 2011. Kathmandu, Nepal: Ministry of Health and Population, New ERA, and ICF International, Calverton, Maryland, 2012.

[18] Ruthstein SO and Johnson K. The DHS Wealth Index. DHS Comparative Report no 6. Calverton, Maryland: ORC Macro, 2004.

[19] Acharya $P$ and Khanal V. The effect of mother's educational status on early initiation of breastfeeding: Further analysis of three consecutive Nepal Demographic and Health Surveys. BMC Public Health 2015; 15: 1069. 